\title{
Significant Parameters in the Optimization of MALDI-TOF-MS for Synthetic Polymers ${ }^{\dagger}$
}

\author{
Stephanie J. Wetzel, ${ }^{*}$ Charles M. Guttman, and Kathleen M. Flynn \\ Polymers Division, National Institute of Standards and Technology, Gaithersburg, Maryland, USA
}

\author{
James J. Filliben \\ Statistical Engineering Division, National Institute of Standards and Technology, Gaithersburg, Maryland, \\ USA
}

\begin{abstract}
One of the most significant issues in any analytical practice is optimization. Optimization and calibration are key factors in quantitation. In matrix-assisted laser desorption/ionization time-of-flight mass spectrometry (MALDI-TOF-MS), instrument optimization is a limitation restricting quantitation. An understanding of the parameters that are most influential and the effects of these parameters on the mass spectrum is required for optimization. This understanding is especially important when characterizing synthetic polymers by MALDI-TOF-MS, due to the breadth of the polymer molecular mass distribution (MMD). Two considerations are important in quantitation, additivity of signal and signal-to-noise $(\mathrm{S} / \mathrm{N})$. In this study, the effects of several instrument parameters were studied using an orthogonal experimental design to understand effects on the signal-to-noise $(\mathrm{S} / \mathrm{N})$ of a polystyrene distribution. The instrument parameters examined included detector voltage, laser energy, delay time, extraction voltage, and lens voltage. Other parameters considered were polymer concentration and matrix. The results showed detector voltage and delay time were the most influential of the instrument parameters for polystyrene using all trans-retinoic acid (RA) as the matrix. These parameters, as well as laser energy, were most influential for the polystyrene with dithranol as the matrix. (J Am Soc Mass Spectrom 2006, 17, 246-252) (c) 2006 American Society for Mass Spectrometry
\end{abstract}

$\mathrm{M}$ any factors influence the quality of matrixassisted laser desorption/ionization (MALDI) time-of flight (TOF) mass spectra of synthetic polymers [1-11]. Foremost is the nature of the sample itself. The chemical composition of the polymer can influence desorption and ionization. For example, it has been amply demonstrated that MALDI-TOF-MS fails to accurately characterize polymers with polydispersities greater than about 1.2 [12-15]. The matrix type and concentrations of the polymer analyte and matrix salt used to prepare the sample have also been shown to influence the polymer distribution obtained by MALDITOF-MS [16-18]. Laser energy has been shown to cause fragmentation in some polymers while often higher mass oligomers require higher laser energies for desorption [9]. Even with a given sample preparation, instrument-toinstrument variation may be significant [19].

Published online January 18, 2006

Address reprint requests to Dr. S. J. Wetzel, Department of Chemistry, Duquesne University, 600 Forbes Avenue, Pittsburgh, PA 15282, USA. E-mail: wetzels@duq.edu

* Current address: Department of Chemistry and Biochemistry, Duquesne University, Pittsburgh, PA.

+ Official Contribution of the National Institute of Standards and Technology; not subject to copyright in the United States.
Such problems are universal to MALDI-TOF-MS users. For example, multiple biomolecules are often quantitated in one mass spectrum, and if their masses vary greatly, this will influence their quantitation. Studying the optimization of polymer samples should help to understand the optimization and quantitation of synthetic polymers as well as biomolecules and organometallics.

In this paper, we have used a factorial design to study various instrumental parameters and sample preparation procedures for optimization of the MALDITOF-MS [20]. Factorial design is an analysis tool that enables many parameters to be considered in one experiment with a minimal number of observations. Also, factorial design allows insight into the effects of parameter interactions that are not evident when considering only one parameter at a time. A factorial design is one in which every setting of every factor appears with every setting of every other factor. Full factorial experiments can require many runs; a carefully chosen fraction of the runs may be all that is necessary. Properly chosen "fractional factorial designs" for 2-level experiments are both balanced and orthogonal. Orthogonal fractional factorial designs enable many parameters to be varied at once, with a minimal number of runs, and are easier to interpret than more complex designs [24]. 
The instrument parameters varied here were detector voltage, laser energy, delay time, extraction voltage, and Einzel lens voltage. Laser energy and delay time are parameters that influence how much sample is desorbed and cationized in the MALDI process. The Einzel lens and extraction voltages influence the ion envelope. The detector voltage influences the sensitivity of the detector, but if the detector voltage is too high, a loss in the signal-to-noise may result because of the increase in background noise. To keep the design relatively simple and the amount of data to a minimum, we chose to concentrate on only two sample preparation parameters: matrix type and polymer concentration [6, $8,10,21]$.

The MALDI-TOF-MS was optimized for analysis of a $9 \mathrm{ku}$ polystyrene (PS) sample using an orthogonal fractional factorial design. At mass $9 \mathrm{ku}$ on our instrument, optimizing resolution is difficult because of mass and isotope broadening. Thus, signal-to-noise $(\mathrm{S} / \mathrm{N})$, instead of resolution, was chosen as the response variable to be studied. Instrumental parameters and their effect on signal-to-noise were the focus of this study, evaluating orthogonal fractional factorial design as an optimization method and identifying significant instrument parameters for polystyrene.

\section{Experimental}

\section{Samples and Reagents}

MALDI-TOF-MS analysis was performed using a $9 \mathrm{ku}$ polystyrene sample that was synthesized by Scientific Polymer Products, Inc. (Ontario, NY). Matrixes used in these experiments were all-trans-retinoic acid and dithranol, both purchased from Aldrich Chemical (Milwaukee, WI) and used as received. Silver trifluoroacetate (AgTFA) (Aldrich Chemical) was used as received. The solvent used for all the experiments was tetrahydrofuran (THF), stabilized with butylhydroxytoluene (BHT) (Mallinckrodt Baker, Inc., Phillipsburg, NJ).

\section{MALDI-TOF-MS}

All experiments were performed on a Bruker (Billerica, MA) Reflex II MALDI-TOF-MS upgraded with a $2 \mathrm{GHz}$ digitizer and the TADS operating system. [Commercial equipment is identified in this article in order to specify adequately the experimental procedure. In no case does such identification imply recommendation or endorsement by the National Institute of Standards and Technology, nor does it imply that the items identified are necessarily the best available for the purpose.] The instrument is equipped with a dual micro channel plate detector for linear mode and a tiltable micro channel plate detector for reflectron mode. A nitrogen laser operating at $337 \mathrm{~nm}$ with a $3 \mathrm{~ns}$ pulse width was utilized. All mass spectra in this experiment were obtained in reflectron mode. The estimated standard uncertainty in the $\mathrm{S} / \mathrm{N}$ is estimated to be 20 .

\section{Sample Preparation}

The two matrixes studied were dithranol and all-transretinoic acid. AgTFA was used for polystyrene cationization. The polymer concentrations studied initially were 1:40:1, 1:80:1, and 1:120:1 by mass of PS:dithranol: AgTFA and 1:75:1, $1: 150: 1$ and $1: 225: 1$ by mass of PS:RA:AgTFA. All samples were deposited onto the MALDI sample target by electrospray to increase signal repeatability and reduce "hot spots" [22]. The samples were electrosprayed using a voltage of $5 \mathrm{kV}$ and a flow rate of $5 \mu \mathrm{L} / \mathrm{min}$. Each mass spectrum was the sum of 100 laser shots and was taken from random spots on the MALDI target. Three signal-to-noise ratios were calculated for each polymer distribution using a low mass peak, a high mass peak, and a peak in the center of the polystyrene molecular mass distribution (MMD). Bruker Xmass software was utilized to calculate the signal-to-noise.

\section{Statistical Analysis Methods}

A $2^{5-1}$ fractional factorial design with triplicated center point was employed to study the optimization of MALDI-TOF-MS. This is a highly efficient $(\mathrm{k}=5$ factor, $n=19$ run) orthogonal design. Orthogonal designs yield precise effect estimates with minimal bias in addition to the parameter effects and interaction effects of any factorial design [23, 24]. The $2^{5-1}$ orthogonal fractional factorial design has many advantages. It requires a minimal number of runs for a given number of parameters. It yields simplified least-squares parameter estimates and statistically unbiased effect estimates. This design also minimizes the uncertainty of the effect estimates, has the ability to estimate both main effects and two term effects, has a simplified confounding structure, and is easy to interpret. Because there are only two values at which each parameter is run, it is easy to interpret parameter effects. The six sample preparation conditions yielded six $2^{5-1}$ designs. The layout for the balanced and orthogonal fractional factorial $2^{5-1}$ design is shown in Table 1 . Our instrument parameters have been applied to the orthogonal $2^{5-1}$ layout. Each design includes 19 spectra at varying instrument parameters, but constant sample preparation parameters.

\section{Sampling Method}

In this study, MALDI-TOF-MS is optimized for a $9 \mathrm{ku}$ polystyrene. We chose to consider only signal-to-noise as a response variable. At lower mass both signal-tonoise and resolution could be used in an optimization experiment. We initially considered both signal-tonoise and the number-average molecular mass $\left(M_{n}\right)$, but the effects on $M_{n}$ will not be discussed in this study. In this paper, we chose to only discuss the signal-tonoise in the evaluation of the optimization method. Further studies will be completed with respect to effects on the $\mathrm{M}_{\mathrm{n}}$. The signal-to-noise was measured at the 
Table 1. The $2^{5-1}$ orthogonal fractional factorial design for Test 1, which is polystyrene at a polymer concentration of 1:75:1 (PS:RA: AgTFA) where "0" represents the midpoint control settings, "+" represents the maximum setting for the parameter, and "-" represents the minimum parameter setting for each parameter. (See Table 2 for the actual settings.)

\begin{tabular}{|c|c|c|c|c|c|c|c|c|}
\hline $\begin{array}{l}\text { Observation } \\
\text { Number }\end{array}$ & $\begin{array}{l}\text { Detector } \\
\text { Voltage }\end{array}$ & $\begin{array}{l}\text { Laser } \\
\text { Energy }\end{array}$ & $\begin{array}{l}\text { Delay } \\
\text { Time }\end{array}$ & $\begin{array}{l}\text { Extraction } \\
\text { Voltage }\end{array}$ & $\begin{array}{c}\text { Lens } \\
\text { Voltage }\end{array}$ & $\begin{array}{c}\mathrm{S} / \mathrm{N} \\
\text { Low mass }\end{array}$ & $\begin{array}{c}\mathrm{S} / \mathrm{N} \\
\text { Center }\end{array}$ & $\begin{array}{c}\mathrm{S} / \mathrm{N} \\
\text { High mass }\end{array}$ \\
\hline 1 & 0 & 0 & 0 & 0 & 0 & 76.9 & 164.2 & 68.6 \\
\hline 2 & - & - & - & - & + & 13 & 15.3 & 11.7 \\
\hline 3 & + & - & - & - & - & 59.2 & 131 & 72.7 \\
\hline 4 & - & + & - & - & - & 10.9 & 11.2 & 10.4 \\
\hline 5 & + & + & - & - & + & 52.2 & 85.1 & 46.7 \\
\hline 6 & - & - & - & + & - & 11.6 & 11.8 & 11.3 \\
\hline 7 & + & - & - & + & + & 84.6 & 175.9 & 71.4 \\
\hline 8 & - & + & - & + & + & 9.8 & 12.3 & 11.6 \\
\hline 9 & + & + & - & + & - & 59.5 & 73.5 & 41.9 \\
\hline 10 & 0 & 0 & 0 & 0 & 0 & 83.3 & 177.5 & 68.4 \\
\hline 11 & - & - & + & - & - & 14.2 & 16.5 & 13.9 \\
\hline 12 & + & - & + & - & + & 453.3 & 1053.8 & 483.1 \\
\hline 13 & - & + & + & - & + & 11.3 & 13.1 & 11.1 \\
\hline 14 & + & + & + & - & - & 214.8 & 416.1 & 198.2 \\
\hline 15 & - & - & + & + & + & 13.2 & 16.3 & 14.6 \\
\hline 16 & + & - & + & + & - & 335.5 & 667.3 & 287.9 \\
\hline 17 & - & + & + & + & - & 11.6 & 11.6 & 11.8 \\
\hline 18 & + & + & + & + & + & 247.8 & 522.4 & 235 \\
\hline 19 & 0 & 0 & 0 & 0 & 0 & 87.1 & 182.2 & 75.7 \\
\hline
\end{tabular}

following three peaks in the distribution; 7500, 8900, and $10,500 \mathrm{u}$. Three peaks were examined over the mass distribution to make sure that no effect of mass existed on the signal-to-noise optimization.

We took data at a minimum value and a maximum value for each parameter, as well as a midpoint value for each parameter (Table 1). For most instrument parameters, the midpoint values were those that were typical for a $9 \mathrm{ku}$ polystyrene in our laboratory, and a higher and lower value was chosen for each. There are only three delay time options on our mass spectrometer; they are short (250 ns), medium (500 ns), and long (750 ns). Table 1 shows the $2^{5-1}$ design that is balanced and orthogonal, and our parameters were applied to this design. The data were taken in random order, so each polymer concentration and matrix change resulted in a different order. This minimizes any bias attributable to instrument drifts with time. Of the 19 spectra collected for each sample preparation parameter, the midpoint control spectra were taken first, last, and midway through (1st, 10th, and 19th spectra). These control spectra are necessary for trend/drift assessment, model validation, and uncertainty assessment. Table 2 shows the actual values of the minimum, maximum, and midpoint for each parameter.

Table 2. Values for the minimum, maximum and midpoint of each parameter in the design.

\begin{tabular}{llccc}
\hline \multicolumn{1}{c}{ Parameter } & & - & 0 & + \\
\hline \hline Detector voltage & $\mathrm{X}_{1}$ & $1.40 \mathrm{kV}$ & $1.55 \mathrm{kV}$ & $1.70 \mathrm{kV}$ \\
Laser energy & $\mathrm{X}_{2}$ & $2.55 \mu \mathrm{J}$ & $3.40 \mu \mathrm{J}$ & $4.50 \mu \mathrm{J}$ \\
Delay time & $\mathrm{X}_{3}$ & Short & Medium & Long \\
Extraction voltage & $\mathrm{X}_{4}$ & $16.2 \mathrm{kV}$ & $17.0 \mathrm{kV}$ & $17.8 \mathrm{kV}$ \\
Lens voltage & $\mathrm{X}_{5}$ & $8.2 \mathrm{kV}$ & $9.0 \mathrm{kV}$ & $9.8 \mathrm{kV}$ \\
\hline
\end{tabular}

\section{Results and Discussion}

In a factorial analysis, mean plots are used to represent the data and provide a first look at the parameter effects. Figure 1 shows the plot of the overall means of each parameter for all the data collected. A first look at the data reveals that the most influential instrument parameters on the signal-to-noise are detector voltage and the time length of the delayed extraction. (It should be noted that Figure 1 and all figures are for the $\mathrm{S} / \mathrm{N}$ at the center mass, $8900 \mathrm{u}$. All three peaks used in the determination of the $\mathrm{S} / \mathrm{N}$ showed the same parameter influences.) In Figure 1, each of the instrument parameters has three data points plotted. The first point is the $\mathrm{S} / \mathrm{N}$ average of the spectra measured for all sample preparations in which the parameter was at its minimum or low value. The second data point is the average $\mathrm{S} / \mathrm{N}$ of the midpoint values, and the third data point is the $\mathrm{S} / \mathrm{N}$ average of the spectra measured maximum or high value of the parameter. The longer delay time also yields higher $\mathrm{S} / \mathrm{N}$ values for this particular polymer. The $\mathrm{S} / \mathrm{N}$ as a function of laser energy, extraction voltage, and lens voltage change slightly over the range of values considered, but their effects are small relative to the detector voltage and the delay length.

The last three plots in Figure 1, test, matrix, and polymer concentration represent means determined by the experimental design. The test represents all six sample sets, comprised of three polymer concentrations in either all trans-retinoic acid or dithranol. The first three test data points represent data obtained in dithranol, and the last three test points are means of signal in RA. The matrix data reveals higher signal-to-noise values in RA, and the polymer concentration yields 


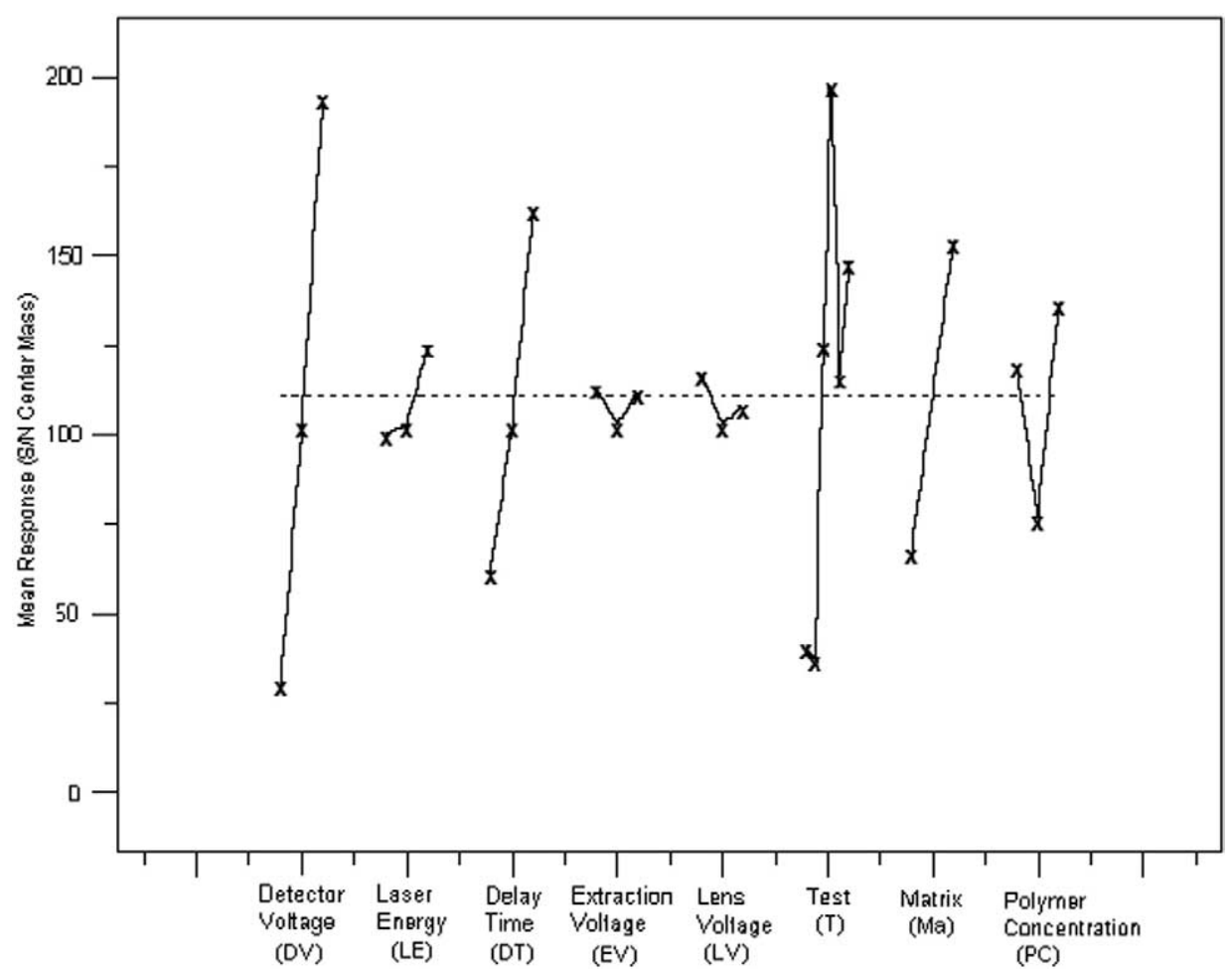

Figure 1. The main effects plot for each parameter for all six tests simultaneously. This plot indicates that detector voltage and delay time are robustly important, regardless of matrix and polymer concentration.

higher mean values of $\mathrm{S} / \mathrm{N}$ at the highest polymer concentration.

For retinoic acid, the highest polymer concentration (1:75:1, PS:RA:AgTFA) yields the highest mean signalto-noise. For dithranol the highest $\mathrm{S} / \mathrm{N}$ is obtained at the lowest polymer concentration (1:120:1, PS:dithranol: AgTFA). A more in-depth analysis was performed on each of these polymer concentrations, where the highest $\mathrm{S} / \mathrm{N}$ was obtained for polystyrene in each matrix.

The main effects plot for polystyrene in retinoic acid is shown in Figure 2. The main effects plot reaffirms that the detector voltage and delay time are the most influential parameters on $\mathrm{S} / \mathrm{N}$. The longest delay time and highest detector voltage yield higher $\mathrm{S} / \mathrm{N}$ values. Laser energy also influences the signal to noise, although not as greatly. Lower laser energies resulted in higher signal-to-noise values. In summary, the optimal settings would be (detector voltage $=+$, laser energy $=$ - , and delay time $=+$ ) with the extraction voltage and lens voltage being of lesser importance.

Interactions occur when the effect of one parameter on a response depends on the level of another parameter. The interaction effects matrix for polystyrene in retinoic acid is shown in Figure 3. The interaction effects matrix is made up of 15 graphs that represent the effect of each individual parameter as well as the interaction of each parameter with every other parameter. Each graph has 2 points, the first point is the mean $\mathrm{S} / \mathrm{N}$ for the low values of that particular parameter, and the second point is mean $\mathrm{S} / \mathrm{N}$ for the high values of the parameter. When an interaction is being considered, the first point in the graph is the mean $\mathrm{S} / \mathrm{N}$ where both parameters have opposite settings $[(+,-)$ or $(-,+)]$, and the second point is the mean $\mathrm{S} / \mathrm{N}$ when both parameters have the same settings $[(+,+)$ or $(-,-)]$. Important parameters or interactions are indicated by steeply sloped lines connecting the two points; a near-zero slope would indicate unimportant parameters and interactions. The parameter identification and its estimated effect Please provide equation is given in the upper left corner of each graph. For example, 1:377.1 in the corner of the first graph in Figure 3 indicates detector voltage increases the signal to noise response on the average of 377.1 units as the detector voltage changes from the low setting $(1.40 \mathrm{kV})$ to the high setting $(1.70 \mathrm{kV})$. Due to the orthogonal backbone of the design, this increase of 377.1 is robust over all of the remaining four parameters under consideration. Again due to the orthogonal design, this estimate of 377.1 is identically the parameter effect least-squares estimate. As seen previously, Figure 3 confirms that the greatest effects on $\mathrm{S} / \mathrm{N}$ are due to detector voltage and delay time. The interaction effects matrix also reveals that the interaction between the detector voltage and the delay time is influential.

The polystyrene analyzed in dithranol has a lower overall $\mathrm{S} / \mathrm{N}$, but reveals similar results to those seen for retinoic acid, with a few exceptions. Although, as seen in Figure 4, both the detector voltage and delay time are also important influences on the $\mathrm{S} / \mathrm{N}$ as was seen in $\mathrm{RA}$, 


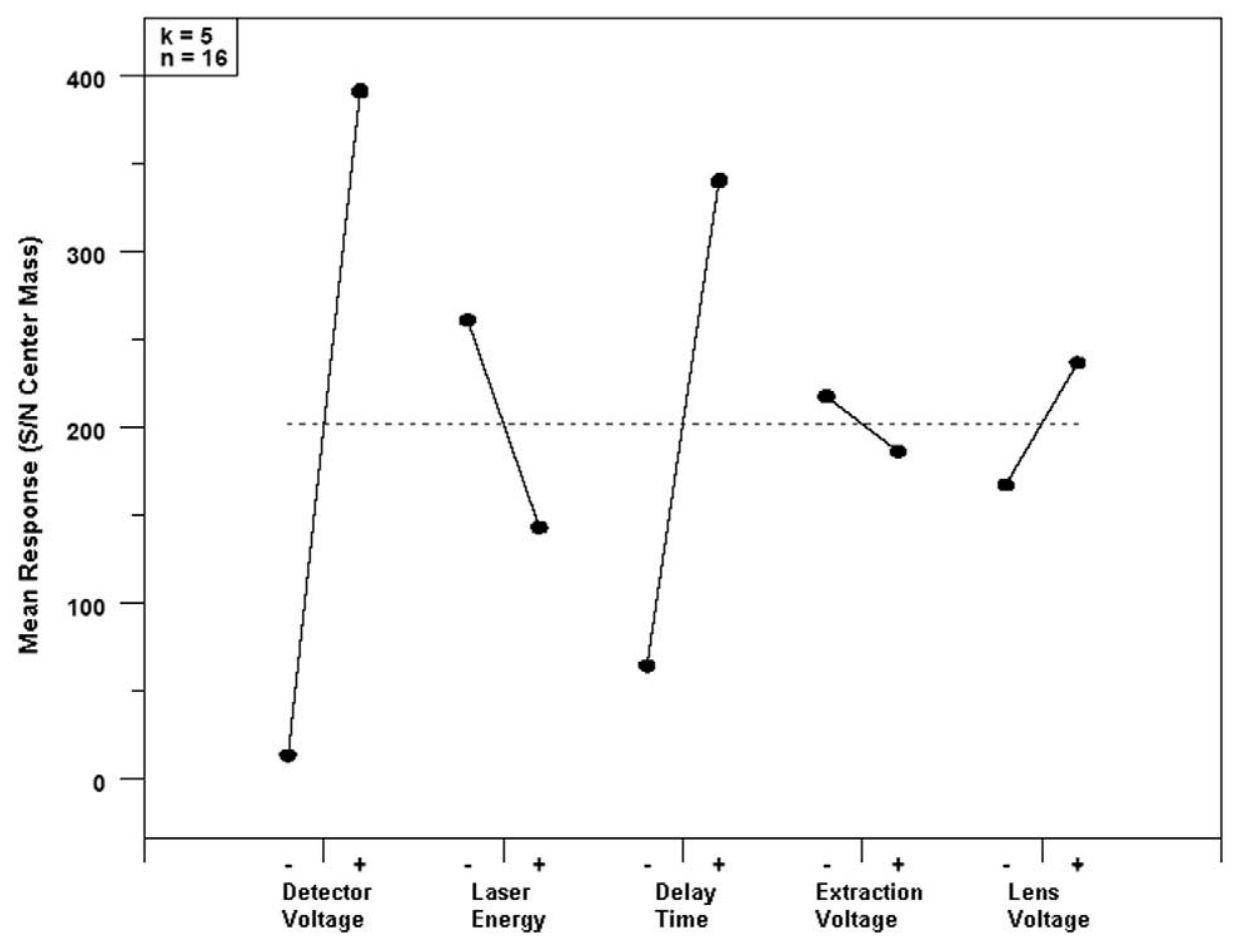

Figure 2. Main effects plot for polystyrene in RA at 1:75:1 polymer concentration.

the main effects plot for polystyrene in dithranol shows a much greater effect of laser energy. When RA was the matrix, lower laser energies yielded higher $\mathrm{S} / \mathrm{N}$ values. But when dithranol was the matrix, higher laser energies yielded higher $\mathrm{S} / \mathrm{N}$. This difference in the effect of laser energy on the $\mathrm{S} / \mathrm{N}$ is attributable to the difference in the matrix properties. Dithranol requires more laser energy than RA to ablate polystyrene into the gas phase [9]. In summary, the best settings for polystyrene in dithranol are (detector voltage $=+$, laser energy $=+$,

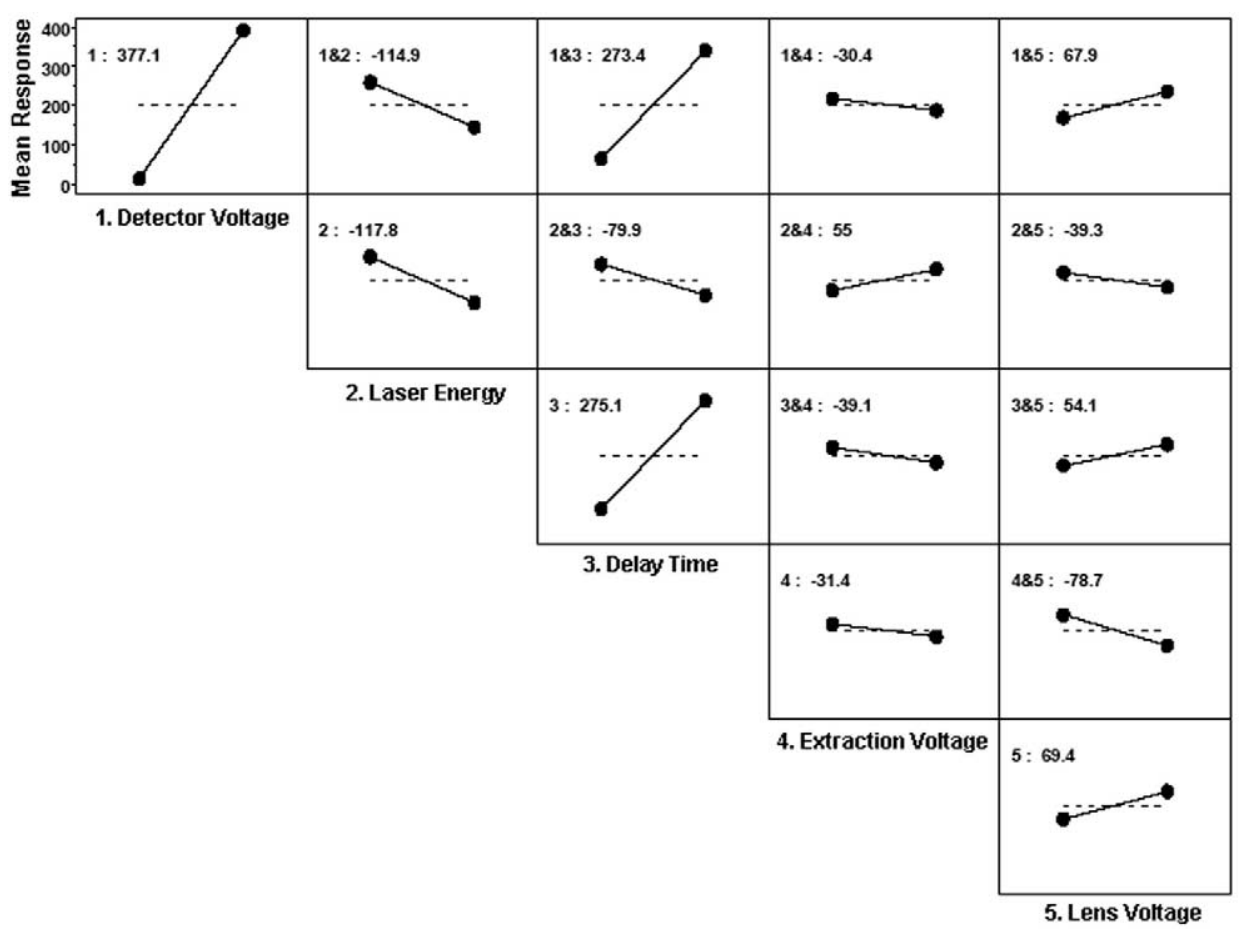

Figure 3. Interaction effects matrix for polystyrene in RA at 1:75:1 polymer concentration. The parameter/interaction and effect estimates are given in the top left corner of each graph. 


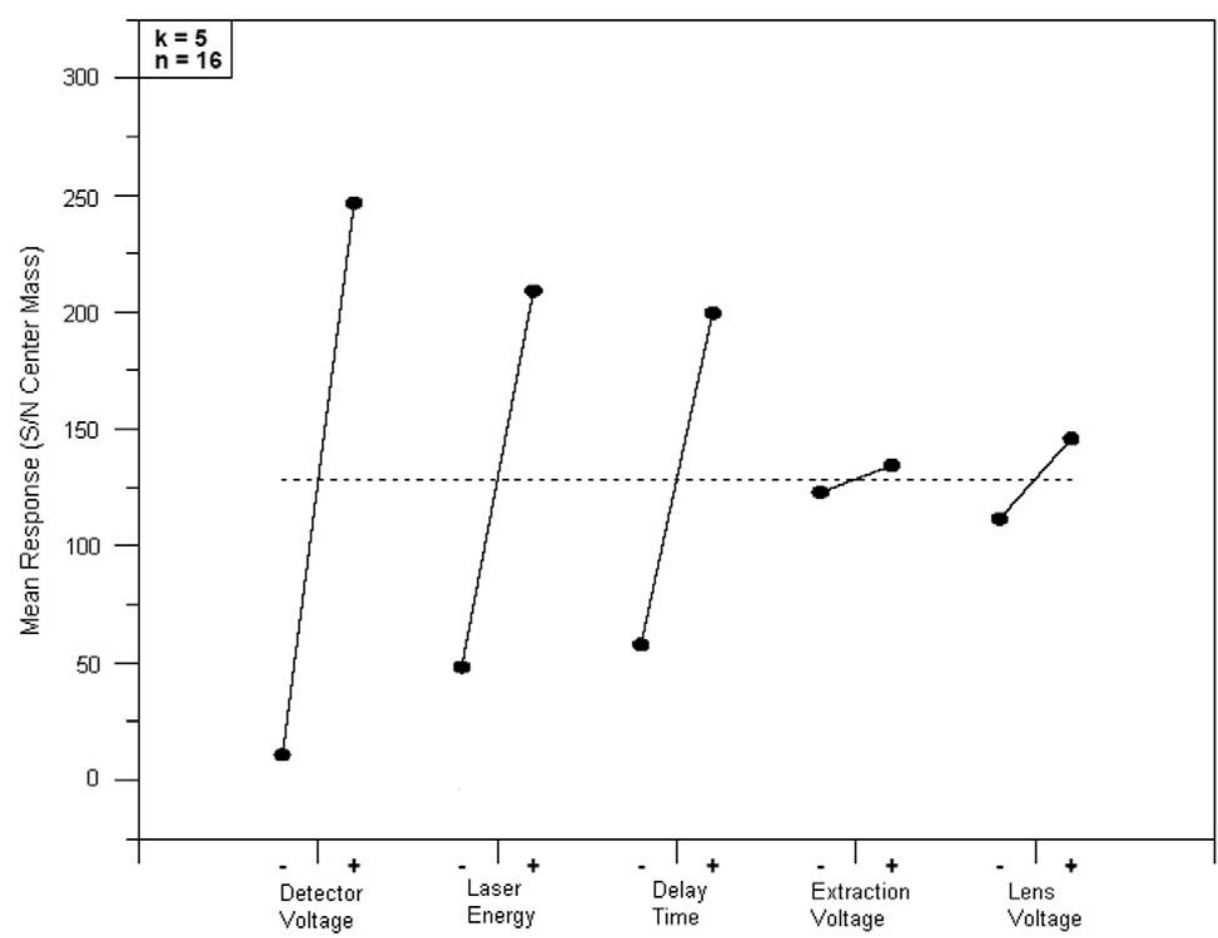

Figure 4. Main effects plot for polystyrene in dithranol at a concentration of 1:15:1. This plot identifies that important parameters are detector voltage, laser energy, and delay time.

and delay time $=+$ ) with lens voltage and extraction voltage being less important.

The interaction effects matrix shown in Figure 5 reveals that for dithranol, many more of the parameters show positive interactions relative to RA. The most influential parameters are still detector voltage and laser energy, but the interaction between these two parameters is also very important. Other significant

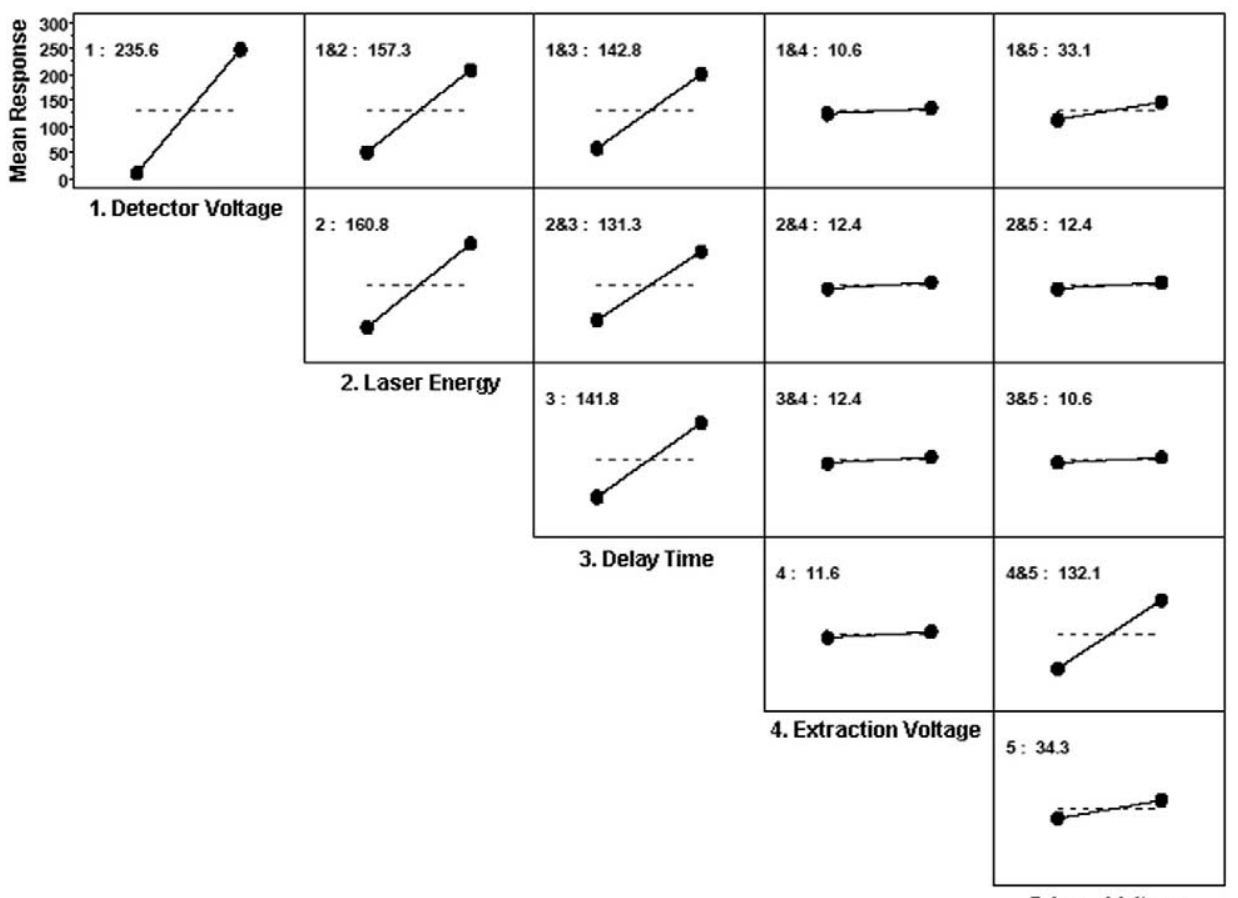

5. Lens Voltage

Figure 5. Interaction effects matrix for polystyrene in dithranol at a concentration of 1:15:1; $\mathrm{S} / \mathrm{N}$ for center mass. 
parameter effects are seen for delay time, the interaction between delay time and laser energy, and the interaction between delay time and detector voltage. In conclusion, the analysis of the polystyrene in dithranol reveals that detector voltage, laser energy, and delay time (along with their 2- and 3-term interactions) are important and extraction voltage and lens voltage are unimportant.

Orthogonal fractional factorial design yields simple plots that are easily interpreted. The minimal amount of data required was easily obtained without an extensive expenditure of time. This study revealed that future optimization studies can eliminate the Einzel lens and extraction voltages, and more sample preparation methods can be added to the design.

\section{Conclusions}

The analysis of polystyrene in RA revealed that the most influential instrument parameters are detector voltage and delay time with higher polymer-to-matrix ratio yielding higher $\mathrm{S} / \mathrm{N}$ values. The analysis of polystyrene in dithranol yielded different conclusions about effects of instrument parameters and sample preparation conditions. The detector voltage and delay time were still influential instrument parameters, but laser energy also significantly influenced the S/N. In dithranol, the lower polymer concentration yielded the highest $\mathrm{S} / \mathrm{N}$ values. Overall, the $\mathrm{S} / \mathrm{N}$ values obtained for RA were higher than those obtained for polystyrene in dithranol. In general, higher detector voltages yield higher $\mathrm{S} / \mathrm{N}$ values and longer delay times also increase $\mathrm{S} / \mathrm{N}$.

The matrix type is a very significant parameter in MALDI sample preparation. The instrument settings that optimize $\mathrm{S} / \mathrm{N}$ differ depending on the matrix type. Optimization is necessary not only for each sample, but also for each matrix. Also, the matrix influences the optimal sample preparation parameters. The polymerto-matrix ratio required for optimization is matrixdependent.

Of the six sample preparation conditions used in this analysis, the two that yielded the highest $\mathrm{S} / \mathrm{N}$ were dithranol at a 1:120:1 concentration and RA at 1:75:1. For polystyrene in dithranol, the best settings were when the detector voltage, laser energy, and delay time were all at their highest settings (detector voltage $=1.70$ $\mathrm{kV}$, laser energy $=4.50 \mu \mathrm{J}$, delay time $=$ long). The optimal settings for polystyrene in RA were when detector voltage and delay time were at their highest settings and laser energy was at its low setting (detector voltage $=$ 1.70 , laser energy $=2.55 \mu \mathrm{J}$, delay time $=$ long).

Orthogonal fractional factorial designs work well for the development of an optimization method in MALDITOF-MS, since many parameters can be considered at the same time without an abundant number of mass spectra required. Integration of this method of analysis into a routine sample preparation technique could improve the repeatability and quantitation capabilities of MALDI-TOF-MS.

\section{References}

1. McEwen, C. N.; Jackson, C.; Larsen, B. S. Instrumental effects in the analysis of polymers of wide polydispersity by MALDI mass spectrometry. Int. J. Mass Spectrom. Ion Processes 1997, 160, 387-394.

2. Zhu, H. H.; Yalcin, T.; Li, L. Analysis of the accuracy of determining average molecular weights of narrow polydispersity polymers by matrix-assisted laser desorption ionization time-of-flight mass spectrometry. J. Am. Soc. Mass Spectrom. 1998, 9, 275-281.

3. Guttman, C. M.; Wetzel, S. J.; Blair, W. R.; Fanconi, B. M.; Girard, J. E.; Goldschmidt, R. J.; Wallace, W. E.; Vanderhart, D. L. NIST-sponsored interlaboratory comparison of polystyrene molecular mass distribution obtained by matrix-assisted laser desorption/ionization time-of-flight mass spectrometry: Statistical analysis. Anal. Chem. 2001, 73, 1252-1262.

4. Bahr, U.; Deppe, A.; Karas, M.; Hillenkamp, F.; Giessmann, U. Mass spectrometry of synthetic polymers by UV-matrix-assisted laser desorption/ionization. Anal. Chem. 1992, 64, 2866-2869.

5. Danis, P. O.; Karr, D. E. A facile sample preparation for the analysis of synthetic organic polymers by matrix-assisted laser desorption/ionization. Org. Mass Spectrom. 1993, 28, 923-925.

6. Schriemer, D. C.; Li, L. A. Mass discrimination in the analysis of polydisperse polymers by MALDI time-of-flight mass spectrometry. 1 . Sample preparation and desorption/ionization issues. Anal. Chem. 1997, 69, 4169-4175.

7. Schriemer, D. C.; Li, L. A. Mass discrimination its the analysis of polydisperse polymers by MALDI time-of-flight mass spectrometry. 2. Instrumental issues. Anal. Chem. 1997, 69, 4176-4183.

8. Wetzel, S. J.; Guttman, C. M.; Flynn, K. M. The influence of electrospray deposition in matrix-assisted laser desorption/ionization mass spectrometry sample preparation for synthetic polymers. Rapid Commun. Mass Spectrom. 2004, 18, 1139-1146.

9. Wetzel, S. J.; Guttman, C. M.; Girard, J. E. The influence of matrix and laser energy on the molecular mass distribution of synthetic polymers obtained by MALDI-TOF-MS. Int. J. Mass Spectrom. 2004, 238(3), 215225.

10. Yalcin, T.; Dai, Y. Q.; Li, L. Matrix-assisted laser desorption/ionization time-of-flight mass spectrometry for polymer analysis: Solvent effect in sample preparation. J. Am. Soc. Mass Spectrom. 1998, 9, 1303-1310.

11. Bauer, B. J.; Byrd, H. C. M.; Guttman, C. M. Small angle neutron scattering measurements of synthetic polymer dispersions in matrixassisted laser desorption/ionization matrixes. Rapid Commun. Mass Spectrom. 2002, 16, 1494-1500.

12. Montaudo, G.; Montaudo, M. S.; Puglisi, C.; Samperi, F. Characterization of polymers by matrix-assisted laser desorption/ionization timeof-flight mass-spectrometry molecular-weight estimates in samples of varying polydispersity. Rapid Commun. Mass Spectrom. 1995, 9, 453-460.

13. Jackson, C.; Larsen, B.; McEwen, C. Comparison of most probable peak values as measured for polymer distributions by MALDI mass spectrometry and by size exclusion chromatography. Anal. Chem. 1996, 68, 1303-1308.

14. Byrd, H. C. M.; McEwen, C. N. The limitations of MALDI-TOF mass spectrometry in the analysis of wide polydisperse polymers. Anal. Chem. 2000, 72, 4568-4576.

15. Montaudo, G.; Montaudo, M. S.; Puglisi, C.; Samperi, F. Molecular weight determination and structural analysis in polydisperse polymers by hyphenated gel permeation chromatography matrix-assisted laser desorption ionization-time of flight mass spectrometry. Int. J. Polym. Anal. Charact. 1997, 3, 177-192.

16. Goldschmidt, R. J.; Guttman, C. M. Response saturation of polystyrene in MALDI. Abstracts. Am. Chem. Soc. 2000, 219, U363.

17. Goldschmidt, R. J.; Owens, K. G. Investigations of the cationization process in the MALDI-TOF-MS analysis of synthetic polymers. Proceedings of the 45th Conference on Mass Spectrometry and Allied Topics; Palm Springs, CA, 1997, p 1106.

18. Gies, A. P.; Nonidez, W. K. MALDI-TOF-MS optimization studies for obtaining mass spectra of poorly soluble/insoluble wholly aromatic polyamides. Proceedings of the 52nd Conference on Mass Spectrometry and Allied Topics; Nashville, TN, May 2004.

19. Guttman, C. M.; Wetzel, S. J.; Flynn, K. M.; Fanconi, B. M.; Van derHart, D. L.; Wallace, W. E. Matrix-assisted laser desorption/ionization timeof-flight mass spectrometry interlaboratory comparison of mixtures of polystyrene with different end groups: Statistical analysis of mass fractions and mass moments. Anal. Chem. 2005, 77, 4539-4548.

20. Kachigan, S. K. Multivariate Statistical Analysis, 2nd ed.; Radius Press: New York, 1991.

21. Owens, K. G. Sample preparation issues in the analysis of synthetic polymers by MALDI-TOF MS. Abstracts. Am. Chem. Soc. 2000, 219, U363-U364.

22. Hanton, S. D.; Hyder, I. Z.; Stets, J. R.; Owens, K. G.; Blair, W. R.; Guttman, C. M.; Giuseppetti, A. A. Investigations of electrospray sample deposition for polymer MALDI mass spectrometry. J. Am. Soc. Mass Spectrom. 2004, 15, 168-179.

23. Prinz, J.; Tobias, P.; Guthrie, W. F.; Hembree, B.; Croarkin, M. C.; Filliben, J. J.; Heckert, N. A. NIST/SEMATECH e-Handbook of Statistical Methods. NIST Handbook 151, Chap V, Section 5, Subsection 9. 2-1-2003.

24. Dey, A. Orthogonal Fractional Factorial Designs; Wiley: New York, 1985, pp. $1-133$. 